\title{
Nonlocal Operational Calculi for Dunkl Operators ${ }^{\star}$
}

\author{
Ivan H. DIMOVSKI and Valentin Z. HRISTOV
}

Institute of Mathematics and Informatics, Bulgarian Academy of Sciences, Acad. G. Bonchev Str., Block 8, 1113 Sofia, Bulgaria

E-mail:dimovski@math.bas.bg,valhrist@bas.bg

Received October 15, 2008, in final form March 04, 2009; Published online March 09, 2009 doi:10.3842/SIGMA.2009.030

\begin{abstract}
The one-dimensional Dunkl operator $D_{k}$ with a non-negative parameter $k$, is considered under an arbitrary nonlocal boundary value condition. The right inverse operator of $D_{k}$, satisfying this condition is studied. An operational calculus of Mikusiński type is developed. In the frames of this operational calculi an extension of the Heaviside algorithm for solution of nonlocal Cauchy boundary value problems for Dunkl functional-differential equations $P\left(D_{k}\right) u=f$ with a given polynomial $P$ is proposed. The solution of these equations in mean-periodic functions reduces to such problems. Necessary and sufficient condition for existence of unique solution in mean-periodic functions is found.
\end{abstract}

Key words: Dunkl operator; right inverse operator; Dunkl-Appell polynomials; convolution; multiplier; multiplier fraction; Dunkl equation; nonlocal Cauchy problem; Heaviside algorithm; mean-periodic function

2000 Mathematics Subject Classification: 44A40; 44A35; 34K06

Here the one-dimensional Dunkl operators $D_{k} f(x)=\frac{d f(x)}{d x}+k \frac{f(x)-f(-x)}{x}, k \geq 0$, in $C^{1}(\mathbb{R})$ under a nonlocal boundary value condition $\Phi\{f\}=0$ with an arbitrary non-zero linear functional $\Phi$ in $C(\mathbb{R})$ are considered. The right inverse operators $L_{k}$ of $D_{k}$, defined by $D_{k} L_{k} f=f$ and $\Phi\left\{L_{k} f\right\}=0$ are studied. To this end, the elements of corresponding operational calculi are developed. A convolution product $f * g$ on $C(\mathbb{R})$, such that $L_{k} f=\{1\} * f$, is found. Further, the convolution algebra $(C(\mathbb{R}), *)$ is extended to its ring $\mathfrak{M}_{k}$ of the multipliers. $(C(\mathbb{R}), *)$ may be conceived as a part of $\mathfrak{M}_{k}$ due to the embedding $f \hookrightarrow f *$. The ring $\mathcal{M}_{k}$ of multiplier fractions $\frac{A}{B}$, such that $A, B \in \mathfrak{M}_{k}$ and $B$ being non-divisor of zero in the operator multiplication, is constructed.

A Heaviside algorithm for effective solution of nonlocal Cauchy boundary value problems for Dunkl functional-differential equations $P\left(D_{k}\right) u=f$ with polynomials $P$ is developed. The solution of these equations in mean-periodic functions reduces to such problems. Necessary and sufficient condition for existence of unique solution in mean-periodic functions is found.

The operational calculus, developed here, is a generalization of the nonlocal operational calculus for $D_{0}=\frac{d}{d x}$ (see Dimovski [6]). Some background material about the Dunkl operators is taken from our previous paper [7] without proofs.

\section{The right inverse operators of $D_{k}$ in $C(\mathbb{R})$ and corresponding Taylor formulae}

Let $L_{k}$ denote an arbitrary right inverse operator of $D_{k}$ in $C(\mathbb{R})$. First, we consider a special right inverse $\Lambda_{k}$ of $D_{k}$, where $y(x)=\Lambda_{k} f(x)$ for $f \in C(\mathbb{R})$ is the solution of the equation $D_{k} y=f(x)$ with initial condition $y(0)=0$.

\footnotetext{
${ }^{\star}$ This paper is a contribution to the Special Issue on Dunkl Operators and Related Topics. The full collection is available at http://www.emis.de/journals/SIGMA/Dunkl_operators.html
} 
Lemma 1. The right inverse operator $\Lambda_{k}$ of $D_{k}$, defined by the initial condition $\Lambda_{k} f(0)=0$ has the form

$$
\Lambda_{k} f(x)=\int_{0}^{x}\left[f_{\mathrm{o}}(t)+\left(\frac{t}{x}\right)^{2 k} f_{\mathrm{e}}(t)\right] d t,
$$

where $f_{\mathrm{e}}$ and $f_{\mathrm{o}}$ are the even and the odd parts of $f$, respectively.

The proof is a matter of a simple check (see [7, p. 198]).

In the general case, an arbitrary right inverse operator $L_{k}$ of $D_{k}$ has a representation of the form

$$
L_{k} f(x)=\int_{0}^{x}\left[f_{\mathrm{e}}(t)+\left(\frac{t}{x}\right)^{2 k} f_{\mathrm{o}}(t)\right] d t+C .
$$

In order $L_{k}$ to be a linear operator, the additive constant $C$ should depend on $f$ and to be a linear functional $\Psi\{f\}$ in $C(\mathbb{R})$. Hence, an arbitrary linear right inverse operator $L_{k}$ of $D_{k}$ in $C(\mathbb{R})$ has the form

$$
L_{k} f(x)=\Lambda_{k} f(x)+\Psi\{f\},
$$

with a linear functional $\Psi$ in $C(\mathbb{R})$.

According to the general theory of right invertible operators (Bittner [2], Przeworska-Rolewicz [12]), an important characteristic of $L_{k}$ is its initial projector

$$
F f(x)=f(x)-L_{k} D_{k} f(x)=\Phi\{f\} .
$$

It maps $C^{1}(\mathbb{R})$ onto ker $D_{k}=\mathbb{C}$, i.e. it is a linear functional $\Phi$ on $C^{1}(\mathbb{R})$. This identity written in the form

$$
L_{k} D_{k} f(x)=f(x)-\Phi\{f\} .
$$

will be used later. Expressing $\Phi$ by $\Psi$, we obtain

$$
\Phi\{f\}=f(0)-\Psi\left\{D_{k} f\right\} .
$$

Let us note that $\Phi\{1\}=1$, which expresses the projector property of $F$.

Considering the right inverse operator $L_{k}$ of $D_{k}$, it is more convenient to look on $L_{k} f=y$ as the solution of an elementary boundary value problem of the form

$$
D_{k} y=f, \quad \Phi\{y\}=0
$$

assuming that $\Phi$ is a given linear functional on $C(\mathbb{R})$ with $\Phi\{1\}=1$. This restriction of the class of right inverse operators $L_{k}$ of $D_{k}$ is adequate when we are to consider nonlocal Cauchy problems for Dunkl equations.

Theorem 1. Let $\Phi: C(\mathbb{R}) \rightarrow \mathbb{C}$ be a linear functional, such that $\Phi\{1\}=1$. Then the right inverse operator $L_{k}$ of $D_{k}$, defined by the boundary value condition $\Phi\left\{L_{k} f\right\}=0$ has the form

$$
L_{k} f(x)=\int_{0}^{x}\left[f_{\mathrm{e}}(y)+\left(\frac{y}{x}\right)^{2 k} f_{\mathrm{o}}(y)\right] d y-\Phi_{t}\left\{\int_{0}^{t}\left[f_{\mathrm{e}}(y)+\left(\frac{y}{t}\right)^{2 k} f_{\mathrm{o}}(y)\right] d y\right\} .
$$

The proof follows immediately from Lemma 1 and the condition $\Phi\{1\}=1$. 
Definition 1. The polynomials

$$
A_{k, n}(x)=L_{k}^{n}\{1\}(x), \quad n=0,1,2, \ldots
$$

are said to be Dunkl-Appell polynomials.

Lemma 2. The Dunkl-Appell polynomials system $\left\{A_{k, n}(x)\right\}_{n=0}^{\infty}$ satisfies the recurrences

$$
A_{k, 0}(x) \equiv 1, \quad \text { and } \quad D_{k} A_{k, n+1}(x)=A_{k, n}(x), \quad \Phi\left\{A_{k, n+1}\right\}=0, \quad n \geq 0
$$

and conversely, (4) implies (3).

The check is immediate. Similar polynomials are introduced implicitly by M. Rösler and M. Voit [14, p. 346].

Lemma 3 (Taylor formula with remainder term). If $f \in C^{(N)}(\mathbb{R})$, then

$$
f(x)=\sum_{j=0}^{N-1} \Phi\left\{D_{k}^{j} f\right\} A_{k, j}(x)+L_{k}^{N}\left(D_{k}^{N} f\right)(x),
$$

where $A_{k, j}(x)=L_{k}^{j}\{1\}(x)$ are Dunkl-Appell polynomials.

This formula is an analogue of the particular case of the Taylor formula known as the Maclaurin formula.

Proof. Delsarte [4], Bittner [2], and Przeworska-Rolewicz [12] give variants of the Taylor formula for right invertible operators in linear spaces. In our case (5) can be written as

$$
I=\sum_{j=0}^{N-1} L_{k}^{j} F D_{k}^{j}+L_{k}^{N} D_{k}^{N}
$$

where $I$ is the identity operator and $F=I-L_{k} D_{k}$. In functional form the above identity takes the form

$$
f(x)=\sum_{j=0}^{N-1} L_{k}^{j} F D_{k}^{j} f(x)+L_{k}^{N} D_{k}^{N} f(x),
$$

where the initial projector $F$ of $L_{k}(1)$ is the linear functional $\Phi$ :

$$
F f(x)=f(x)-L_{k} D_{k} f(x)=\Phi\{f\} .
$$

$F$ projects the space $C(\mathbb{R})$ onto the space $\mathbb{C}$ of the constants. Hence

$$
f(x)=\sum_{j=0}^{N-1} \Phi\left\{D_{k}^{j} f\right\} L_{k}^{j}\{1\}(x)+L_{k}^{N} D_{k}^{N} f(x),
$$

which is the Taylor formula (5). 


\section{Convolutional products for the right inverses $L_{k}$ of $D_{k}$}

In Dunkl [8, Theorem 5.1] the similarity operator

$$
V_{k} f(x)=b_{k} \int_{-1}^{1} f(x y)(1-y)^{k-1}(1+y)^{k} d y, \quad b_{k}=\frac{\Gamma(2 k+1)}{2^{2 k} \Gamma(k) \Gamma(k+1)}
$$

is found, which transforms the differentiation operator $D=\frac{d}{d x}$ into $D_{k}$ :

$$
V_{k} D=D_{k} V_{k}
$$

Usually this operator is called intertwining operator. The constant $b_{k}$ is chosen to ensure that $V_{k}\{1\}=1$.

The problem of inverting the Dunkl intertwining operator $V_{k}$ is discussed by several authors, see e.g. Trimèche [15], Betankor, Sifi, Trimèche [1], but we will use the explicit formulae from Ben Salem and Kallel [3, p. 159].

Denoting $S f(x)=\frac{1}{2 x} \frac{d f(x)}{d x}$, the inverse $V_{k}^{-1}$ of $V_{k}$ has the following representations:

(i) If $k=n+r$ is non-integer with integer part $n$ and $r \in(0,1)$, then

$$
\begin{aligned}
V_{k}^{-1} f(x)= & c_{k}\left[|x| S^{n+1}\left\{\int_{0}^{|x|}\left(x^{2}-y^{2}\right)^{-r} f_{\mathrm{e}}(y) y^{2 k} d y\right\}\right. \\
& \left.+\operatorname{sign}(x) S^{n+1}\left\{\int_{0}^{|x|}\left(x^{2}-y^{2}\right)^{-r} f_{\mathrm{o}}(y) y^{2 k+1} d y\right\}\right], \quad x \neq 0,
\end{aligned}
$$

where $c_{k}=\frac{2 \sqrt{\pi}}{\Gamma\left(n+r+\frac{1}{2}\right) \Gamma(1-r)}$.

(ii) If $k$ is a non-negative integer, then

$$
V_{k}^{-1} f(x)=\frac{\sqrt{\pi}}{\Gamma\left(k+\frac{1}{2}\right)}\left[x S^{k}\left(x^{2 k-1} f_{\mathrm{e}}(x)\right)+S^{k}\left(x^{2 k} f_{\mathrm{o}}(x)\right)\right], \quad x \neq 0 .
$$

$V_{k}$ transforms $C(\mathbb{R})$ into a proper subspace $\widetilde{C_{k}}=V_{k}(C(\mathbb{R}))$ of it. $V_{k}$ is a similarity from a right inverse operator $\Lambda$ of $D_{0}=\frac{d}{d x}$ to $L_{k}$. In order to specify the operator $\Lambda$ let us define the linear functional

$$
\widetilde{\Phi}\{f\}=\left(\Phi \circ V_{k}\right)\{f\}
$$

in $\widetilde{C_{k}}$. Then define $\Lambda: \widetilde{C_{k}} \rightarrow \widetilde{C_{k}}$ to be the solution $y=\Lambda \widetilde{f}$ of the elementary boundary value problem

$$
D_{0} y(x) \equiv y^{\prime}(x)=\widetilde{f}(x), \quad \widetilde{\Phi}\{y\}=0 .
$$

This solution has the form

$$
\Lambda \widetilde{f}(x)=\int_{0}^{x} \widetilde{f}(y) d y-\widetilde{\Phi_{t}}\left\{\int_{0}^{t} \widetilde{f}(\tau) d \tau\right\} .
$$

Lemma 4. The following similarity relation holds

$$
V_{k} \Lambda=L_{k} V_{k} .
$$


Proof. Applying $V_{k}$ to the defining equation $D(\Lambda \widetilde{f})=\tilde{f}$, one obtains

$$
V_{k} D(\Lambda \widetilde{f})=V_{k} \tilde{f}=f \quad \text { or } \quad D_{k}\left(V_{k} \Lambda \widetilde{f}\right)=V_{k} \tilde{f}=f .
$$

In fact, the boundary value condition $\widetilde{\Phi}\{\Lambda \widetilde{f}\}=0$ can be written as $\Phi\left\{V_{k} \Lambda \widetilde{f}\right\}=0$. Hence $u=V_{k} \Lambda \tilde{f}$ is the solution of the boundary value problem $D_{k} u=f, \Phi\{u\}=0$, i.e. $u=L_{k} f$. Therefore

$$
V_{k} \Lambda V_{k}^{-1} f=L_{k} f \quad \text { or } \quad V_{k} \Lambda=L_{k} V_{k} .
$$

The similarity relation (4) allows to introduce a convolution structure $*: C(\mathbb{R}) \times C(\mathbb{R}) \rightarrow$ $C(\mathbb{R})$, such that $L_{k}$ to be the convolution operator $L_{k}=\{1\} *$ in $C(\mathbb{R})$.

The operator $\Lambda$ is defined not only in $\widetilde{C_{k}}$, but in the whole space $C(\mathbb{R})$. This allows to introduce a convolution structure $\widetilde{*}: C(\mathbb{R}) \times C(\mathbb{R}) \rightarrow C(\mathbb{R})$.

Lemma 5. The operation

$$
(\widetilde{f} \widetilde{*})(x)=\widetilde{\Phi}_{t}\left\{\int_{t}^{x} \widetilde{f}(x+t-\tau) \widetilde{g}(\tau) d \tau\right\}
$$

is a bilinear, commutative and associative operation in $\widetilde{C_{k}}=V_{k}(C(\mathbb{R}))$ such that

$$
\Lambda \widetilde{f}=\{1\} \widetilde{*} \widetilde{f} .
$$

It satisfies the boundary value condition $\widetilde{\Phi}\{\widetilde{f} \widetilde{*} \widetilde{g}\}=0$ for arbitrary $\widetilde{f}$ and $\widetilde{g}$ in $C(\mathbb{R})$.

The proof of the assertion that $\widetilde{f} \widetilde{g}$ is an inner operation in $\widetilde{C_{k}}$ follows directly from the explicit inversion formula for $V_{k}$ (see Xu [16] or Ben Salem and Kallel [3, Theorem 1.1]). In Dimovski [5, p. 52] it is proved that (6) is a bilinear, commutative and associative operation in $C(\mathbb{R})$, and hence in $\widetilde{C_{k}}=V_{k}(C(\mathbb{R}))$. The second relation $(7)$ is obvious. The proof of $\widetilde{\Phi}\{\widetilde{f} \widetilde{g}\}=0$ is also elementary (see Dimovski [5, p. 54]).

Theorem 2. The operation

$$
f * g=D_{k}^{2 n} V_{k}\left[\left(V_{k}^{-1} L_{k}^{n} f\right) \widetilde{*}\left(V_{k}^{-1} L_{k}^{n} g\right)\right],
$$

where $n$ is the integer part of $k$, is a convolution of $L_{k}$ in $C(\mathbb{R})$ such that

$$
L_{k} f=\{1\} * f
$$

and the boundary value condition $\Phi\{f * g\}=0$ is satisfied for arbitrary $f$ and $g$ in $C(\mathbb{R})$.

Proof. The assertion of the theorem follows from Lemmas 5 and 4 and a general theorem of Dimovski [5, Theorem 1.3.6, p. 26]. This convolution is introduced in Dimovski, Hristov and Sifi [7].

Remark 1. The convolution (8) reduces to

$$
f * g=V_{k}\left[\left(V_{k}^{-1} f\right) \widetilde{*}\left(V_{k}^{-1} g\right)\right]
$$

for $n=0$, i.e. when $0<k<1$.

From (9) and Definition 1 it follows that

$$
L_{k}^{N+1} f=\left\{A_{k, N}\right\} * f,
$$

where $A_{k, N}$ is the Dunkl-Appell polynomial of degree exactly $N$. This allows also to state the Taylor formula (5) with remainder term in the Cauchy form:

Lemma 6. If $f \in C^{(N)}(\mathbb{R})$, then

$$
f(x)=\sum_{j=0}^{N-1} \Phi\left\{D_{k}^{j} f\right\} A_{k, j}(x)+\left(A_{k, N-1} * D_{k}^{N} f\right)(x),
$$

where $A_{k, j}(x), j=0,1,2, \ldots, N-1$, are the Dunkl-Appell polynomials $A_{k, j}(x)=L_{k}^{j}\{1\}$. 


\section{The ring of multipliers of the convolutional algebra $(C(\mathbb{R}), *)$}

The convolutional algebras $(C(\mathbb{R}), *)$ with convolution product $(8)$, are annihilators-free (or algebras without order in the terminology of Larsen [9, p. 13]). This means that in each of these algebras $f * g=0, \forall g \in C(\mathbb{R})$, implies $f=0$.

Definition 2. An operator $A: C(\mathbb{R}) \rightarrow C(\mathbb{R})$ is said to be a multiplier of the convolutional algebra $(C(\mathbb{R}), *)$ iff

$$
A(f * g)=(A f) * g
$$

for arbitrary $f, g \in C(\mathbb{R})$.

As it is shown in Larsen [9], it is not necessary to assume neither that $A$ is a linear operator, nor that it is continuous in $C(\mathbb{R})$. These properties of the multipliers follow automatically from (10). Something more, a general result of Larsen [9, p. 13] implies

Theorem 3. The set of the multipliers of the convolutional algebra $(C(\mathbb{R}), *)$ form a commutative ring $\mathfrak{M}_{k}$.

The simplest multipliers of $(C(\mathbb{R}), *)$ are the numerical operators $[\alpha]$ for $\alpha \in \mathbb{C}$, defined by

$$
[\alpha] f=\alpha f, \quad \forall f \in C(\mathbb{R}),
$$

and the convolutional operators $f *$ for $f \in C(\mathbb{R})$, defined by

$$
(f *) g=f * g, \quad \forall g \in C(\mathbb{R}) .
$$

Further we need the following characterization result for the multipliers of $(C(\mathbb{R}), *)$ :

Theorem 4. A linear operator $A: C(\mathbb{R}) \rightarrow C(\mathbb{R})$ is a multiplier of $(C(\mathbb{R}), *)$ iff it admits a representation of the form

$$
\text { Af }=D_{k}(m * f),
$$

where the function $m=A\{1\}$ is such that $m * f \in C^{1}(\mathbb{R})$ for all $f \in C(\mathbb{R})$.

Proof. Let $A: C(\mathbb{R}) \rightarrow C(\mathbb{R})$ be a multiplier of $(C(\mathbb{R}), *)$. The operator $L_{k} f=\{1\} * f$ is also a multiplier. Then, according to Theorem 3,

$$
A L_{k}=L_{k} A \text {. }
$$

Applying $A$ to $L_{k} f=\{1\} * f$, we get

$$
L_{k} A f=A L_{k} f=A(\{1\} * f)=(A\{1\}) * f .
$$

The identity

$$
L_{k}(A f)=m * f
$$

with $m=A\{1\}$ is possible only if $m * f \in C^{1}(\mathbb{R})$ for each $f \in C(\mathbb{R})$. It remains to apply $D_{k}$ to (12) in order to obtain (11).

Conversely, let $A: C(\mathbb{R}) \rightarrow C(\mathbb{R})$ be the operator defined by (11), i.e. $A f=D_{k}(m * f)$, where $m \in C(\mathbb{R})$ is such that $m * f \in C^{1}(\mathbb{R})$ for all $f \in C(\mathbb{R})$. Then

$$
A(f * g)=D_{k}(m *(f * g))=D_{k}((m * f) * g) .
$$

But $m * f=L_{k} D_{k}(m * f)$ due to formula $(2)$ since $\Phi(m * f)=0$ by Theorem 2 . Then

$$
A(f * g)=D_{k} L_{k}\left[D_{k}(m * f) * g\right]=(A f) * g .
$$

Hence $A$ is a multiplier of the convolution algebra $(C(\mathbb{R}), *)$. 
The specification of the function $m=A\{1\}$ is, in general, a nontrivial problem even in the case of the simplest Dunkl operator $D_{0}=\frac{d}{d x}$ (the usual differentiation). This could be confirmed by the following two examples:

Example 1. If $\Phi\{f\}=f(0)$, then $m$ is a continuous function of locally bounded variation, i.e. $m \in B V \cap C(\mathbb{R})$ (see Dimovski [5, p. 26]).

Example 2. Let $\Phi\{f\}=\int_{0}^{1} f(x) d x$. Then $m \in C(\mathbb{R})$ can be arbitrary (see Dimovski [5, p. 69]).

\section{Nonlocal operational calculi for $D_{k}$}

Our aim here is to develop a direct operational calculus for solution of the following nonlocal Cauchy problem for the operator $D_{k}$ : Solve the equation $P\left(D_{k}\right) u=f$ with a polynomial $P$ and a given $f \in C(\mathbb{R})$ under the boundary value conditions $\Phi\left\{D_{k}^{j} u\right\}=\alpha_{j}, j=0,1,2, \ldots, \operatorname{deg} P-1$, where $\alpha_{j}$ are given constants and $\Phi$ is a nonzero linear functional on $C(\mathbb{R})$.

This is a special case of the problems considered by R. Bittner [2] and D. PrzeworskaRolewicz [12] for an arbitrary right invertible operator $D$ instead of $D_{k}$.

Our intention here is to propose constructive results and to obtain an explicit solution of the boundary value problems considered. This is done by means of an operational calculus essential part of which is an extension of the Heaviside algorithm.

This operational calculus is developed using a direct algebraic approach based on the convolution (8). Instead of Mikusiński's method [10] of convolutional fractions $\frac{f}{g}$, we follow an alternative approach of multiplier fractions $\frac{A}{B}$, where $A$ and $B$ are multipliers of the convolutional algebra $(C(\mathbb{R}), *)$ and $B$ is a non-divisor of zero in the operator multiplication.

Let us consider the ring $\mathfrak{M}_{k}$ of the multipliers of the convolutional algebra $(C(\mathbb{R})$, *). The correspondence $\alpha \mapsto[\alpha]$ is an embedding of $\mathbb{C}$ into $\mathfrak{M}_{k}$. The correspondence $f \mapsto f *$ is an embedding of $(C(\mathbb{R}), *)$ in $\mathfrak{M}_{k}$. Hence, we may consider $\mathbb{C}$ and $C(\mathbb{R})$ as parts of $\mathfrak{M}_{k}$.

$\mathfrak{M}_{k}$ is a commutative ring (Theorem 3 ). The subset $\mathfrak{N}_{k}$ of $\mathfrak{M}_{k}$, consisting of the non-zero non-divisors of zero with respect to the operator multiplication in $\mathfrak{M}_{k}$, is nonempty. Indeed, at least the identity operator $I$ and the right inverse $L_{k}$ of $D_{k}$ belong to $\mathfrak{N}_{k}$. In addition, $\mathfrak{N}_{k}$ is a multiplicative subset, i.e. if $A, B \in \mathfrak{N}_{k}$, then $A B \in \mathfrak{N}_{k}$.

Consider the Cartesian product

$$
\mathfrak{M}_{k} \times \mathfrak{N}_{k}=\left\{(A, B): A \in \mathfrak{M}_{k}, B \in \mathfrak{N}_{k}\right\}
$$

and introduce the equivalence relation

$$
(A, B) \sim\left(A^{\prime}, B^{\prime}\right) \Leftrightarrow A B^{\prime}=B A^{\prime} .
$$

Definition 3. The set $\mathcal{M}_{k}=\mathfrak{M}_{k} \times \mathfrak{N}_{k} / \sim$ obtained by the factorization of $\mathfrak{M}_{k} \times \mathfrak{N}_{k}$ with respect to the equivalence relation (13) is said to be the ring of multiplier fractions.

$\mathcal{M}_{k}$ may be considered both as an extension of the field $\mathbb{C}$ of the complex numbers and of the ring $(C(\mathbb{R}), *)$. Formally, this is seen by the embeddings

$$
\alpha \hookrightarrow \frac{[\alpha]}{I} \quad \text { and } \quad f \hookrightarrow \frac{f *}{I} .
$$

In the sequel we denote the identity operator $I$ simply by 1 . The multiplication operation of the two elements $p$ and $q$ in $\mathcal{M}_{k}$ will be denoted simply by $p q$. Therefore, instead of $f * g$ we will write $f g$. 
For our aims the most important elements of $\mathcal{M}_{k}$ are

$$
L_{k}=\{1\} \quad \text { and } \quad S_{k}=\frac{1}{L_{k}} .
$$

The fraction $S_{k}$ with the identity operator as numerator and with $L_{k}$ as denominator will be called algebraic Dunkl operator. Its relation to the ordinary Dunkl operator $D_{k}$ is given by the following theorem:

Theorem 5. Let $f \in C^{1}(\mathbb{R})$. Then

$$
D_{k} f=S_{k} f-\Phi\{f\} .
$$

Note that identity (14) should be interpreted as

$$
\left(D_{k} f\right) *=S_{k}(f *)-[\Phi\{f\}]
$$

where $\left(D_{k} f\right) *$ and $(f *)$ are to be understood as convolution operators and $[\Phi\{f\}]$ as the numerical operator determined by the number $\Phi\{f\}$. $S_{k}$ is neither convolutional nor numerical operator, but an element of $\mathcal{M}_{k}$.

Proof. In Section 1 (equality (2)) we have seen that

$$
L_{k} D_{k} f=f-\Phi\{f\}
$$

where $\Phi\{f\}$ is the corresponding constant function $\{\Phi\{f\}\}$. Considered as an operator identity, this can be written as $\left(L_{k} D_{k} f\right) *=f *-\{\Phi\{f\}\} *$ or $L_{k}\left[D_{k}(f *)\right]=f *-\Phi\{f\} L_{k}$. Hence

$$
L_{k}\left(D_{k} f\right) *=(f *)-\Phi\{f\} L_{k} .
$$

It remains to multiply by $S_{k}$ to obtain (14).

Relation (14) may be characterized as the basic formula of our operational calculus. Using it repeatedly, we obtain

Corollary 1. Let $f \in C^{(N)}(\mathbb{R})$. Then

$$
D_{k}^{N} f=S_{k}^{N} f-\sum_{j=0}^{N-1} \Phi\left\{D_{k}^{j} f\right\} S_{k}^{N-j-1} .
$$

Remark 2. The last formula is equivalent to the Taylor formula (5) in Section 1.

Let $P(\lambda)=a_{0} \lambda^{m}+a_{1} \lambda^{m-1}+\cdots+a_{m-1} \lambda+a_{m}, a_{0} \neq 0$, and $\Phi$ be a non-zero linear functional on $C(\mathbb{R})$.

Definition 4. The problem for solving the Dunkl functional-differential equation

$$
P\left(D_{k}\right) u=f, \quad f \in C(\mathbb{R})
$$

under the boundary value conditions

$$
\Phi\left\{D_{k}^{j} u\right\}=\alpha_{j}, \quad j=0,1,2, \ldots, m-1
$$

is called a nonlocal Cauchy problem determined by the functional $\Phi$. 
By means of (14) and (15) it is possible to "algebraize" any nonlocal Cauchy boundary value problem.

The simplest nonlocal Cauchy problem for $D_{k}$, determined by a linear functional $\Phi$ in $C(\mathbb{R})$ concerns the functional-differential equation

$$
D_{k} u(x)-\lambda u(x)=f(x)
$$

with the boundary condition $\Phi\{u\}=0$.

It is known that the solution of the homogeneous equation

$$
D_{k} u(x)-\lambda u(x)=0
$$

under the initial condition $u(0)=1$ is

$$
u_{k}(\lambda x)=j_{k-\frac{1}{2}}(i \lambda x)+\frac{\lambda x}{2 k+1} j_{k+\frac{1}{2}}(i \lambda x)
$$

(see Ben Salem and Kallel [3, p. 161]), where $j_{\alpha}(x)$ denotes the modified (normalized) Bessel function

$$
j_{\alpha}(x)=2^{\alpha} \Gamma(\alpha+1) \frac{J_{\alpha}(x)}{x^{\alpha}}, \quad x \neq 0 \quad \text { and } \quad j_{\alpha}(0)=1 .
$$

We introduce the Dunkl indicatrix of the functional $\Phi$ as the following entire function of exponential type:

$$
E_{k}(\lambda)=\Phi_{\xi}\left\{u_{k}(\lambda \xi)\right\}=\Phi_{\xi}\left\{j_{k-\frac{1}{2}}(i \lambda \xi)+\frac{\lambda \xi}{2 k+1} j_{k+\frac{1}{2}}(i \lambda \xi)\right\} .
$$

Lemma 7. The function $\frac{u_{k}(\lambda x)}{E_{k}(\lambda)}$ is the generating function of the Dunkl-Appell polynomials system, i.e.

$$
\frac{u_{k}(\lambda x)}{E_{k}(\lambda)}=\sum_{n=0}^{\infty} \lambda^{n} A_{k, n}(x) .
$$

Here we will skip the simple proof. The linear operator $L_{k, \lambda}$ defined as the solution $u(x)=$ $L_{k, \lambda} f(x)$ of the nonlocal Cauchy boundary value problem

$$
D_{k} u-\lambda u=f, \quad \Phi\{u\}=0,
$$

is said to be the resolvent operator of the Dunkl operator under the boundary value condition $\Phi\{u\}=0$.

Theorem 6. The resolvent operator $L_{k, \lambda}$ admits the convolutional representation

$$
L_{k, \lambda} f(x)=l_{k}(\lambda, x) * f(x), \quad \text { where } \quad l_{k}(\lambda, x)=\frac{u_{k}(\lambda x)}{E_{k}(\lambda)} .
$$

Proof. We will use the formula

$$
D_{k}(f * g)=\left(D_{k} f\right) * g+\Phi\{f\} g
$$

which is true under the assumption $f \in C^{1}(\mathbb{R})$. It follows from a more general result of Dimovski [5, Theorem 1.38], but in our case it can be verified directly. It gives

$$
D_{k}\left\{l_{k}(\lambda, x) * f(x)\right\}=D_{k} l_{k}(\lambda, x) * f(x)+\Phi_{\xi}\left\{l_{k}(\lambda, \xi)\right\} f(x)=\lambda\left\{l_{k}(\lambda, x) * f(x)\right\}+f(x) .
$$

Hence $u=\left\{l_{k}(\lambda, x) * f(x)\right\}$ satisfies the equation $D_{k} u-\lambda u=f$. It remains to verify the boundary value condition $\Phi\{u\}=0$. But it follows from the basic property $\Phi\{f * g\}=0$ of the convolution (Theorem 2). 
The resolvent operator $L_{k, \lambda}$ exists for each $\lambda$ with $E_{k}(\lambda) \neq 0$. The zeros of $E_{k}(\lambda)$ are the eigenvalues of the boundary value problem $D_{k} u-\lambda u=0, \Phi\{u\}=0$. They form an enumerable set $\left\{\lambda_{1}, \lambda_{2}, \ldots, \lambda_{n}, \ldots\right\}$ except in the case when $\Phi$ is a Dirac functional $\Phi\{f\}=f(a)$, when $E_{k}(\lambda) \neq 0$ for all $\lambda \in \mathbb{C}$.

It is easy to find the solution of our problem in $\mathcal{M}_{k}$. Using the basic formula of the operational calculus (see Theorem 5), we have $D_{k} u=S_{k} u$ since $\Phi\{u\}=0$, and then

$$
S_{k} u-\lambda u=f \quad \text { or } \quad\left(S_{k}-\lambda\right) u=f .
$$

In order to write the solution

$$
u=\frac{1}{S_{k}-\lambda} f
$$

we must be sure that $S_{k}-\lambda$ is non-divisor of zero.

Lemma 8. $S_{k}-\lambda$ is a divisor of zero in $\mathcal{M}_{k}$ iff $E_{k}(\lambda)=0$.

Proof. Let $S_{k}-\lambda$ be a divisor of zero in $\mathcal{M}_{k}$. Then there exists a multiplier fraction $\frac{A}{B}$ such that $A \neq 0$ and

$$
\left(S_{k}-\lambda\right) \frac{A}{B}=0,
$$

which is equivalent to $\left(S_{k}-\lambda\right) A=0$. Since $A \neq 0$, then there is a function $g \in C(\mathbb{R})$ such that $A g=v \neq 0$. Then

$$
\left(S_{k}-\lambda\right) v=0 .
$$

Multiplying by $L_{k}$ we get

$$
\left(1-\lambda L_{k}\right) v=0 \quad \text { or } \quad v-\lambda L_{k} v=0 .
$$

Since $\Phi\left(L_{k} v\right)=0$ by the definition of $L_{k}$ (Section 1 ), then $\Phi\{v\}=0$.

Applying $D_{k}$, we get $D_{k} v-\lambda v=0, \Phi\{v\}=0$. According to Ben Salem and Kallel [3], all the non-zero solutions of $D_{k} v-\lambda v=0$ are $v=C\left(j_{k-\frac{1}{2}}(i \lambda x)+\frac{\lambda x}{2 k+1} j_{k+\frac{1}{2}}(i \lambda x)\right)$ with a constant $C \neq 0$. The boundary value condition $\Phi\{v\}=0$ is equivalent to $E_{k}(\lambda)=0$.

Conversely, if $E_{k}(\lambda)=0$, then there exists a solution $v \neq 0$ of the eigenvalue problem $D_{k} v-\lambda v=0, \Phi\{v\}=0$. For this $v$ we have

$$
\left(S_{k}-\lambda\right) v=0
$$

and hence $S_{k}-\lambda$ is a divisor of zero in $\mathcal{M}_{k}$.

Theorem 7. Let $\lambda \in \mathbb{C}$ be such that $E_{k}(\lambda) \neq 0$. Then

$$
\frac{1}{S_{k}-\lambda}=\left\{l_{k}(\lambda, x)\right\} *=\frac{1}{E_{k}(\lambda)}\left\{j_{k-\frac{1}{2}}(i \lambda x)+\frac{\lambda x}{2 k+1} j_{k+\frac{1}{2}}(i \lambda x)\right\} * .
$$

Proof. We have seen that

$$
L_{k, \lambda} f(x)=\left\{l_{k}(\lambda, x)\right\} * f .
$$

But for the solution $u=L_{k, \lambda} f$ of the boundary value problem $D_{k} u-\lambda u=f, \Phi\{u\}=0$, in the case $E_{k}(\lambda) \neq 0$ we found

$$
u=\frac{1}{S_{k}-\lambda} f .
$$

Since the convolution $*$ is annihilators-free, then (16) follows from the identity

$$
\frac{1}{S_{k}-\lambda} f=\left\{l_{k}(\lambda, x)\right\} * f .
$$


Corollary 2. If $E_{k}(\lambda) \neq 0$, then

$$
\frac{1}{\left(S_{k}-\lambda\right)^{m}}=\left\{\frac{1}{(m-1) !} \frac{\partial^{m-1}}{\partial \lambda^{m-1}} l_{k}(\lambda, x)\right\} * .
$$

\section{$5 \quad$ Heaviside algorithm for solving nonlocal Cauchy problems for Dunkl operators}

Now we are to apply the elements of the operational calculus developed in the previous section to effective solution of nonlocal Cauchy boundary value problems of the form

$$
P\left(D_{k}\right) u=f, \quad \Phi\left(D_{k}^{j} u\right)=\alpha_{j}, \quad j=0,1,2, \ldots, \operatorname{deg} P-1,
$$

with given $\alpha_{j} \in \mathbb{C}$.

To this end we extend the classical Heaviside algorithm, which is intended for solving initial value problems for ordinary linear differential equations with constant coefficients to the case of Dunkl functional-differential equations.

The extended Heaviside algorithm starts with the algebraization of problem (17). It reduces the problem to a single algebraic equation of the first degree in $\mathcal{M}_{k}$.

Let $P(\lambda)=a_{0} \lambda^{m}+a_{1} \lambda^{m-1}+\cdots+a_{m-1} \lambda+a_{m}$ be a given polynomial of $m$-th degree, i.e. with $a_{0} \neq 0$.

The consecutive steps of the algorithm are the following:

1) Factorize $P(\lambda)$ in $\mathbb{C}$ to

$$
P(\lambda)=a_{0}\left(\lambda-\mu_{1}\right)^{\varkappa_{1}}\left(\lambda-\mu_{2}\right)^{\varkappa_{2}} \cdots\left(\lambda-\mu_{s}\right)^{\varkappa_{s}},
$$

where $\mu_{1}, \mu_{2}, \ldots, \mu_{s}$ are the distinct zeros of $P(\lambda)$ and $\varkappa_{1}, \varkappa_{2}, \ldots, \varkappa_{s}$ are their corresponding multiplicities.

2) Represent each of the terms of the equation by the algebraic Dunkl operator $S_{k}$. This is done by the formulae

$$
D_{k}^{j} u=S_{k}^{j} u-S_{k}^{j-1} \alpha_{0}-S_{k}^{j-2} \alpha_{1}-\cdots-S_{k} \alpha_{j-2}-\alpha_{j-1}, \quad j=1,2, \ldots, m .
$$

Thus we obtain the following equation in $\mathcal{M}_{k}$ :

$$
P\left(S_{k}\right) u=f+Q\left(S_{k}\right), \quad \operatorname{deg} Q<\operatorname{deg} P,
$$

with

$$
Q\left(S_{k}\right)=\sum_{j=0}^{m-1} \sum_{l=0}^{m-j-1} a_{j} \alpha_{l} S_{k}^{m-j-l-1}=\sum_{\mu=0}^{m-1}\left(\sum_{\nu=0}^{m-\mu-1} a_{\nu} \alpha_{m-\mu-\nu-1}\right) S_{k}^{\mu} .
$$

3) Verify if $P\left(S_{k}\right)$ is a non-divisor of zero in $\mathcal{M}_{k}$ by checking if $E_{k}\left(\mu_{j}\right) \neq 0$ for all $j=1,2, \ldots, s$.

4) If $P\left(S_{k}\right)$ is a non-divisor of zero, then write the solution $u$ in $\mathcal{M}_{k}$ :

$$
u=\frac{1}{P\left(S_{k}\right)} f+\frac{Q\left(S_{k}\right)}{P\left(S_{k}\right)}
$$

5) Expand $\frac{1}{P\left(S_{k}\right)}$ and $\frac{Q\left(S_{k}\right)}{P\left(S_{k}\right)}$ into partial fractions:

$$
\frac{1}{P\left(S_{k}\right)}=\sum_{j=1}^{s} \sum_{l=1}^{\varkappa_{j}} \frac{A_{j, l}}{\left(S_{k}-\mu_{j}\right)^{l}}, \quad \frac{Q\left(S_{k}\right)}{P\left(S_{k}\right)}=\sum_{j=1}^{s} \sum_{l=1}^{\varkappa_{j}} \frac{B_{j, l}}{\left(S_{k}-\mu_{j}\right)^{l}} .
$$


6) Interpret the partial fractions as convolution operators

$$
\begin{aligned}
& \frac{1}{S_{k}-\mu_{j}}=\left\{l_{k}\left(\mu_{j}, x\right)\right\} *=\frac{1}{E_{k}\left(\mu_{j}\right)}\left\{j_{k-\frac{1}{2}}\left(i \mu_{j} x\right)+\frac{\mu_{j} x}{2 k+1} j_{k+\frac{1}{2}}\left(i \mu_{j} x\right)\right\} * . \\
& \frac{1}{\left(S_{k}-\mu_{j}\right)^{l}}=\left\{\left.\frac{1}{(l-1) !} \frac{\partial^{l-1}}{\partial \lambda^{l-1}} l_{k}(\lambda, x)\right|_{\lambda=\mu_{j}}\right\} *, \quad l=2,3, \ldots
\end{aligned}
$$

7) Write the convolutional representation

$$
u(x)=(G * f)(x)+R(x), \quad \text { where } \quad G=\frac{1}{P\left(S_{k}\right)}, \quad R=\frac{Q\left(S_{k}\right)}{P\left(S_{k}\right)} .
$$

Example 3. Let $P(\lambda)$ has only simple zeros $\mu_{1}, \mu_{2}, \ldots, \mu_{m}$. Then

$$
\frac{1}{P\left(S_{k}\right)}=\sum_{j=1}^{m} \frac{1}{P^{\prime}\left(\mu_{j}\right)} \cdot \frac{1}{S_{k}-\mu_{j}}=\left\{\sum_{j=1}^{m} \frac{1}{P^{\prime}\left(\mu_{j}\right)} l_{k}\left(\mu_{j}, x\right)\right\} *
$$

and

$$
\frac{Q\left(S_{k}\right)}{P\left(S_{k}\right)}=\sum_{j=1}^{m} \frac{Q\left(\mu_{j}\right)}{P^{\prime}\left(\mu_{j}\right)} \cdot \frac{1}{S_{k}-\mu_{j}}=\left\{\sum_{j=1}^{m} \frac{Q\left(\mu_{j}\right)}{P^{\prime}\left(\mu_{j}\right)} l_{k}\left(\mu_{j}, x\right)\right\} * .
$$

Then the solution $u$ takes the functional form

$$
u(x)=\sum_{j=1}^{m} \frac{1}{P^{\prime}\left(\mu_{j}\right)} l_{k}\left(\mu_{j}, x\right) * f(x)+\sum_{j=1}^{m} \frac{Q\left(\mu_{j}\right)}{P^{\prime}\left(\mu_{j}\right)} l_{k}\left(\mu_{j}, x\right) .
$$

The result of this section can be summarized in the following

Theorem 8. The nonlocal Cauchy problem (Definition 4) for a Dunkl equation $P\left(D_{k}\right) u=f$ has a unique solution in $C^{(m)}(\mathbb{R}), m=\operatorname{deg} P$, iff none of the zeros of the polynomial $P(\lambda)$ is a zero of the indicatrix $E_{k}(\lambda)$, i.e. when

$$
\{\lambda: P(\lambda)=0\} \cap\left\{\lambda: E_{k}(\lambda)=0\right\}=\varnothing .
$$

Remark 3. The term "nonlocal" should not be understood literally. The assertion of Theorem 8 is true also when $\Phi$ is a Dirac functional, i.e. $\Phi\{f\}=f(a)$ for $a \in \mathbb{R}$. For us the most interesting is the case $\Phi\{f\}=f(0)$. Then $E_{k}(\lambda) \equiv 1$ and from the theorem it follows that the initial value problem

$$
P\left(D_{k}\right) u=f, \quad u(0)=\alpha_{0}, \quad\left(D_{k} u\right)(0)=\alpha_{1}, \quad \ldots, \quad\left(D_{k}^{n-1} u\right)(0)=\alpha_{n-1},
$$

always has a unique solution. We will use this fact in the following section.

\section{Mean-periodic functions for $D_{k}$ determined by a linear functional and mean periodic solutions of Dunkl equations}

The notion of mean-periodic function for the differentiation operator $\frac{d}{d t}$, determined by a linear functional $\Phi$ in $C(\mathbb{R})$, is introduced by J. Delsarte [4]:

A function $f \in C(\mathbb{R})$ is said to be mean-periodic with respect to the functional $\Phi$ if it satisfies identically the condition

$$
\Phi_{\tau}\{f(t+\tau)\}=0 .
$$


In order to define mean-periodic functions for the Dunkl operator $D_{k}$ we need to recall the definition of the Dunkl translation (shift) operators, introduced by M. Rösler [13] and later studied in M.A. Mourou and K. Trimèche [11]. They are a class of operators $M: C(\mathbb{R}) \rightarrow C(\mathbb{R})$ commuting with $D_{k}$ in $C^{1}(\mathbb{R})$.

Definition 5. Let $f \in C(\mathbb{R})$ and $y \in \mathbb{R}$. Then $\left(T_{k}^{y} f\right)(x)=u(x, y) \in C^{1}\left(\mathbb{R}^{2}\right)$ is the solution of the boundary value problem

$$
D_{k, x} u(x, y)=D_{k, y} u(x, y), \quad u(x, 0)=f(x) .
$$

$T_{k}^{y}$ is called the translation operator for the Dunkl operator $D_{k}$.

Such a solution exists for arbitrary $f \in C(\mathbb{R})$ and it has the following explicit form (see e.g. $[13,3])$ :

$$
\begin{aligned}
T_{k}^{y} f(x)= & \frac{\Gamma\left(k+\frac{1}{2}\right)}{\Gamma(k) \Gamma\left(\frac{1}{2}\right)}\left[\int_{0}^{\pi} f_{\mathrm{e}}\left(\sqrt{x^{2}+y^{2}-2|x y| \cos t}\right) h^{\mathrm{e}}(x, y, t) \sin ^{2 k-1} t d t\right. \\
& \left.+\int_{0}^{\pi} f_{\mathrm{o}}\left(\sqrt{x^{2}+y^{2}-2|x y| \cos t}\right) h^{\mathrm{o}}(x, y, t) \sin ^{2 k-1} t d t\right] .
\end{aligned}
$$

As usually, the subscripts "e" and "o" denote correspondingly the even and the odd part of a function: $f_{\mathrm{e}}(x)=\frac{f(x)+f(-x)}{2}, f_{\mathrm{o}}(x)=\frac{f(x)-f(-x)}{2}$. As for $h^{\mathrm{e}}(x, y, t)$ and $h^{\mathrm{o}}(x, y, t)$, they denote respectively

$$
\begin{aligned}
& h^{\mathrm{e}}(x, y, t)=1-\operatorname{sign}(x y) \cos t, \\
& h^{\mathrm{o}}(x, y, t)= \begin{cases}\frac{(x+y)(1-\operatorname{sign}(x y) \cos t)}{\sqrt{x^{2}+y^{2}-2|x y| \cos t}} & \text { for }(x, y) \neq(0,0), \\
0 & \text { otherwise. }\end{cases}
\end{aligned}
$$

Lemma 9. The translation operators satisfy the following basic relations:

(i) $\quad T_{k}^{y} f(x)=T_{k}^{x} f(y)$,

(ii) $\quad T_{k}^{y} T_{k}^{z} f(x)=T_{k}^{z} T_{k}^{y} f(x)$,

(iii) $\quad D_{k, x} T_{k}^{y} f(x)=T_{k}^{y} D_{k, x} f(x)$.

Proofs can be found in various publications, in particular, in our paper [7].

A natural extension of the notion of mean-periodic function for the Dunkl operator is proposed by Ben Salem and Kallel [3]. Instead of (18) they use the condition

$$
\Phi_{y}\left\{T_{k}^{y} f(x)\right\}=0
$$

to define mean-periodic function $f$ for $D_{k}$ with respect to the functional $\Phi$. Here $T_{k}^{y}$ is the generalized translation operator just defined.

The space of mean-periodic functions for the Dunkl operator $D_{k}$ with respect to a given functional $\Phi$ will be denoted by $\mathcal{P}_{\Phi}$. We skip the subscript $k$ for sake of simplicity.

Lemma 10. If $f \in \mathcal{P}_{\Phi}$, then $L_{k} f \in \mathcal{P}_{\Phi}$.

Proof. Denote $\varphi(x)=\Phi_{t}\left\{T_{k}^{t} L_{k} f(x)\right\}$ and use the commutation relation (21) from Lemma 9 $D_{k, x} T_{k}^{y} f(x)=T_{k}^{y} D_{k, x} f(x)$ to obtain

$$
D_{k} \varphi(x)=\Phi_{t}\left\{D_{k} T_{k}^{t} L_{k} f(x)\right\}=\Phi_{t}\left\{T_{k}^{t} D_{k} L_{k} f(x)\right\}=\Phi_{t}\left\{T_{k}^{t} f(x)\right\}=0 .
$$

Hence $\varphi(x)=C=$ const. But $\varphi(0)=\Phi_{t}\left\{T_{k}^{t} L_{k} f(0)\right\}=\Phi_{t}\left\{T_{k}^{0} L_{k} f(t)\right\}=\Phi_{t}\left\{L_{k} f(t)\right\}=0$. Hence $C=0$. 
Further we will be interested in the solvability of Dunkl differential-difference equations

$$
P\left(D_{k}\right) u=f
$$

with a polynomial $P$ in the space of the mean-periodic functions $\mathcal{P}_{\Phi}$, defined by (22). We intend also to propose an algorithm for obtaining such solutions.

To this end we are to develop an operational calculus for $D_{k}$ in $C(\mathbb{R})$ and to extend the Heaviside algorithm for it. The following result plays a basic role in the application of this algorithm for solution of Dunkl equations in mean-periodic functions.

Theorem 9. The class of mean-periodic functions $\mathcal{P}_{\Phi}$ is an ideal in the convolutional algebra $(C(\mathbb{R}), *)$, i.e. if $f \in \mathcal{P}_{\Phi}$ and $g \in C(\mathbb{R})$, then $f * g \in \mathcal{P}_{\Phi}$.

Proof. Assume that $f \in \mathcal{P}_{\Phi}$, i.e.

$$
\Phi_{t}\left\{T_{k}^{t} f(x)\right\}=0 .
$$

From Lemma 10 it follows that $L_{k}^{n+1} f \in \mathcal{P}_{\Phi}$ for $n=0,1,2, \ldots$, i.e.

$$
\Phi_{t}\left\{T_{k}^{t} L_{k}^{n+1} f(x)\right\}=0 .
$$

Since $L_{k} f=\{1\} * f$, then $L_{k}^{n+1} f=A_{k, n} * f$, where the Dunkl-Appell polynomial $A_{k, n}$ is of degree exactly $n$. We have

$$
\Phi_{t}\left\{T_{k}^{t}\left(A_{k, n} * f\right)(x)\right\}=0
$$

and then we can assert that

$$
\Phi_{t}\left\{T_{k}^{t}(P * f)(x)\right\}=0
$$

for any polynomial $P$. By an approximation argument it follows that

$$
\Phi_{t}\left\{T_{k}^{t}(g * f)(x)\right\}=0
$$

for arbitrary $g \in C(\mathbb{R})$, i.e. that $g * f \in \mathcal{P}_{\Phi}$.

Corollary 3. Let $M: C(\mathbb{R}) \rightarrow C(\mathbb{R})$ be an arbitrary multiplier of the algebra $(C(\mathbb{R}), *)$. Then $M\left(\mathcal{P}_{\Phi}\right) \subset \mathcal{P}_{\Phi}$, i.e. the restriction of $M$ to $\mathcal{P}_{\Phi}$ is an inner operator in $\mathcal{P}_{\Phi}$.

Proof. Let $f \in \mathcal{P}_{\Phi}$. According to Theorem 4, $M f=D_{k}(m * f)$ with $m=M\{1\}$, then, by Theorem $9, m * f \in \mathcal{P}_{\Phi} \cap C^{1}(\mathbb{R})$. Then $D_{k}(m * f) \in \mathcal{P}_{\Phi}$, i.e. $f \in \mathcal{P}_{\Phi}$ implies $M f \in \mathcal{P}_{\Phi}$.

In the sequel we study the problem of solution of Dunkl equations in mean-periodic functions determined by a linear functional.

Theorem 10. A function $u \in \mathcal{P}_{\Phi} \cap C^{(m)}(\mathbb{R})$ is a solution of the Dunkl equation $P\left(D_{k}\right) u=f$, with $f \in \mathcal{P}_{\Phi}$ iff $u$ is a solution of the homogeneous nonlocal Cauchy problem

$$
P\left(D_{k}\right) u=f, \quad \Phi\left\{D_{k}^{j} u\right\}=0, \quad j=0,1,2, \ldots, m-1, \quad m=\operatorname{deg} P .
$$

Proof. The condition $f \in \mathcal{P}_{\Phi}$ is necessary for the existence of a solution $u \in \mathcal{P}_{\Phi}$. Assume that a function $u \in \mathcal{P}_{\Phi} \cap C^{(m)}(\mathbb{R})$ is a solution of the Dunkl equation $P\left(D_{k}\right) u=f$. Then mean-periodic are all the functions $D_{k}^{j} u, j=0,1,2, \ldots, m-1$, i.e.

$$
\Phi_{y}\left\{T_{k}^{y} D_{k}^{j} u(x)\right\}=0,
$$


since the operator $A f(x)=\Phi_{y}\left\{T_{k}^{y} f(x)\right\}$ commutes with $D_{k}$ (Dimovski, Hristov and Sifi [7]). For $x=0$ from (24) we get

$$
\Phi_{y}\left\{T_{k}^{y} D_{k}^{j} u(0)\right\}=0 .
$$

But $T_{k}^{y} D_{k}^{j} u(0)=T_{k}^{0} D_{k}^{j} u(y)((19)$, Lemma 9) and hence

$$
\Phi\left\{D_{k}^{j} u\right\}=0, \quad j=0,1,2, \ldots, m-1 .
$$

In order to prove that a solution $u$ of $P\left(D_{k}\right) u=f$ with $f \in \mathcal{P}_{\Phi}$, which satisfies conditions (25), is a mean-periodic function, we consider the function

$$
v=\Phi_{y}\left\{T_{k}^{y} u(x)\right\}=A u .
$$

Since the operator $A$ commutes with $D_{k}$, then applying it on the equation $P\left(D_{k}\right) u=f$, we get $P\left(D_{k}\right) v=0$ due to $A f=0$. It remains to find the initial values $D_{k}^{j} v(0), j=0,1,2, \ldots, m-1$ :

$$
D_{k}^{j} v(0)=A D_{k}^{j} u(0)=\Phi_{y}\left\{T_{k}^{y} D_{k}^{j} u(0)\right\}=\Phi_{y}\left\{T_{k}^{0} D_{k}^{j} u(y)\right\}=\Phi_{y}\left\{D_{k}^{j} u(y)\right\}=0 .
$$

At the end of the previous section we have seen that the initial value problem $P\left(D_{k}\right) v=0$, $D_{k}^{j} v(0)=0, j=0,1,2, \ldots, m-1$, has only the trivial solution $v(x)=0$. Thus we proved that $\Phi_{y}\left\{T_{k}^{y} u\right\}=0$, i.e. $u$ is mean-periodic.

Now we can use operational calculus method for solving nonlocal Cauchy problems for Dunkl equations to find explicitly the mean-periodic solutions of such equations.

To this end, we are to solve the homogeneous nonlocal Cauchy boundary value problem

$$
P\left(D_{k}\right) u=f, \quad \Phi\left\{D_{k}^{j} u\right\}=0, \quad j=0,1,2, \ldots, m-1,
$$

with $f \in \mathcal{P}_{\Phi}$.

In the ring $\mathcal{M}_{k}$ of the multiplier fractions it reduces to the single algebraic equation for $u$

$$
P\left(S_{k}\right) u=f .
$$

As we have seen in Section 4, $P\left(S_{k}\right)$ is a non-divisor of zero in $\mathcal{M}_{k}$ iff none of the zeros of the polynomial $P(\lambda)$ is a zero of the Dunkl indicatrix $E_{k}(\lambda)$. If $P\left(S_{k}\right)$ is a divisor of zero, then, in order to ensure the existence of solution of (27) and thus of (26), additional restrictions on $f$ should be imposed. This is the so called resonance case, which we will not treat here.

Thus, let $P\left(S_{k}\right)$ be a non-divisor of zero in $\mathcal{M}_{k}$, i.e. $\{\lambda: P(\lambda)=0\} \cap\left\{\lambda: E_{k}(\lambda)=0\right\}=\varnothing$. Then the formal solution of $(27)$ in $\mathcal{M}_{k}$

$$
u=\frac{1}{P\left(S_{k}\right)} f
$$

can be written in explicit functional form. Using the extended Heaviside algorithm of Section 5, we represent $\frac{1}{P\left(S_{k}\right)}$ as a convolutional operator

$$
\frac{1}{P\left(S_{k}\right)}=\{G(x)\} *
$$

Then

$$
u=G * f
$$

is the desired mean-periodic solution of the Dunkl equation $P\left(D_{k}\right) u=f$. The verification is straightforward. Indeed, $G * f \in \mathcal{P}_{\Phi}$ according to Theorem 9 , since $f \in \mathcal{P}_{\Phi}$.

Our considerations of the problem for solving Dunkl equations in mean-periodic functions can be summarized in the following 
Theorem 11. A Dunkl equation $P\left(D_{k}\right) u=f$ with $f \in \mathcal{P}_{\Phi}$ has a unique solution in $\mathcal{P}_{\Phi}$ iff none of the zeros of the polynomial $P(\lambda)$ is a zero of the Dunkl indicatrix

$$
E_{k}(\lambda)=\Phi\left\{j_{k-\frac{1}{2}}(i \lambda x)+\frac{\lambda x}{2 k+1} j_{k+\frac{1}{2}}(i \lambda x)\right\}
$$

In the end, it is possible the Duhamel principle to be extended to the problem for solving Dunkl equations in mean-periodic functions.

Theorem 12. Let $H(x)$ be the solution of the homogeneous nonlocal Cauchy problem $P\left(D_{k}\right) H=1$, $\Phi\left\{D_{k}^{j} H\right\}=0, j=0,1,2, \ldots, m-1$. Then

$$
u=D_{k}(H * f)
$$

is a mean-periodic solution of the Dunkl equation $P\left(D_{k}\right) u=f$ with $f \in \mathcal{P}_{\Phi}$.

\section{Acknowledgments}

The authors are very grateful to the editors and to the referees for the constructive and valuable comments and recommendations.

\section{References}

[1] Betancor J.J., Sifi M., Trimèche K., Intertwining operator and the commutators of the Dunkl operator on $\mathbb{C}$, Math. Sci. Res. J. 10 (2006), no. 3, 66-78.

[2] Bittner R., Operational calculus in linear spaces, Studia Math. 20 (1961), 1-18.

[3] Ben Salem N., Kallel S., Mean-periodic functions associated with the Dunkl operators, Integral Transforms Spec. Funct. 15 (2004), 155-179.

[4] Delsarte J., Les fonctions moyenne-périodiques, J. Math. Pures Appl. 14 (1935), 403-453.

[5] Dimovski I.H., Convolutional calculus, Kluwer Academic Publishers Group, Dordrecht, 1990.

[6] Dimovski I.H., Nonlocal operational calculi, Proc. Steklov Inst. Math. 203 (1995), no. 3, 53-65.

[7] Dimovski I.H., Hristov V.Z., Sifi M., Commutants of the Dunkl operators in C(R), Fract. Calc. Appl. Anal. 9 (2006), 195-213.

[8] Dunkl C.F., Differential-difference operators associated to reflection groups, Trans. Amer. Math. Soc. 311 (1989), 167-183.

[9] Larsen R., An introduction to the theory of multipliers, Springer-Verlag, New York - Heidelberg, 1971.

[10] Mikusiński J., Operational calculus, I, Warszawa, 1967.

[11] Mourou M.A., Trimèche K., Opérateurs de transmutation et théorème de Paley-Wiener associés à un opérateur aux dérivées et différences sur $\mathbb{R}$, C. R. Acad. Sci. Paris Ser. I Math. 332 (2001), 397-400.

[12] Przeworska-Rolewicz D., Algebraic theory of right inverse operators, Studia Math. 48 (1973), $129-144$.

[13] Rösler M., Bessel-type signed hypergroups on $\mathbb{R}$, in Probability Measures on Groups and Related Structures XI (Oberwolfach, 1994), World Sci. Publ., River Edge, NJ, 1995, 292-304.

[14] Rösler M., Voit M., Biorthogonal polynomials associated with reflection groups and a formula of Macdonald, J. Comput. Appl. Math. 99 (1998), 337-351, q-alg/9711004.

[15] Trimèche K., The Dunkl intertwining operator on spaces of functions and distributions and integral representation of its dual, Integral Transforms Spec. Funct. 12 (2001), 349-374.

[16] Xu Y., An integral formula for generalized Gegenbauer polynomials and Jacobi polynomials, Adv. in Appl. Math. 29 (2002), 328-341. 